\title{
EchoGéo
}

$34 \mid 2015$

Varia

\section{Le Sud et ses fictions. À propos des nouvelles de Eudora Welty}

Entretien avec Jean-Marc Victor, réalisé à Paris le 24 septembre 2015

Jean-Marc Victor et Serge Weber

\section{OpenEdition}

\section{Journals}

Édition électronique

URL : https://journals.openedition.org/echogeo/14417

DOI : 10.4000/echogeo.14417

ISSN : 1963-1197

Éditeur

Pôle de recherche pour l'organisation et la diffusion de l'information géographique (CNRS UMR 8586)

\section{Référence électronique}

Jean-Marc Victor et Serge Weber, "Le Sud et ses fictions. À propos des nouvelles de Eudora Welty », EchoGéo [En ligne], 34 | 2015, mis en ligne le 15 décembre 2015, consulté le 01 août 2021. URL : http:// journals.openedition.org/echogeo/14417 ; DOI : https://doi.org/10.4000/echogeo.14417

Ce document a été généré automatiquement le 1 août 2021.

EchoGéo est mis à disposition selon les termes de la licence Creative Commons Attribution - Pas d'Utilisation Commerciale - Pas de Modification 4.0 International (CC BY-NC-ND) 


\section{Le Sud et ses fictions. À propos des nouvelles de Eudora Welty}

Entretien avec Jean-Marc Victor, réalisé à Paris le 24 septembre 2015

Jean-Marc Victor et Serge Weber

1 Jean-Marc Victor est spécialiste de la littérature américaine. Sa thèse portait sur «l'esthétique de la trace » dans l'œuvre de la romancière, nouvelliste, photographe et essayiste américaine Eudora Welty, dont il est un spécialiste reconnu (1999 ; 2009a). Il a ensuite exploré et fréquenté des textes d'auteurs assez proches comme Flannery O'Connor (Bellehigue, Victor, 2004). En parallèle, il a défriché un autre chantier de recherche, l'analyse de photographies, en particulier celles qui représentent le Sud des États-Unis, «Vieux Sud» ou «Sud profond ( Deep South) : d'abord celles de Eudora Welty elle-même (2009b), ensuite celles de Ralph Eugene Meatyard (2009c; 2010). Toujours dans le sillage de l'univers de Welty, il explore depuis peu un espace bien particulier dans la littérature, celui du jardin, notamment en proposant une nouvelle traduction d'une nouvelle de Virginia Woolf et, plus largement, en proposant de mettre en regard modalités d'écriture de la fiction et modèles botaniques.

2 - Serge Weber (SW). Jean-Marc Victor, vous portez un regard sur des textes qui font une large place à la question de l'espace, de sa représentation et de son traitement littéraire en plus d'avoir interrogé le Deep South en tant qu'espace bien particulier dans l'histoire et la géographie des États-Unis. De plus, vous avez aiguisé votre regard sur la photographie et par là-même creusé la question du rapport entre texte et photographie dans la représentation de l'espace, sans vous éloigner du Vieux Sud. Vos travaux font donc écho à des préoccupations de plus en plus prégnantes en géographie concernant la représentation de l'espace, en particulier celles qui tournent autour des méthodologies visuelles, du statut géographique de textes littéraires et des méthodologies mixtes de recherche qui visent à saisir ce qu'il y a à la fois de sensible, de matériel, de non-représentable et de socialement construit dans le rapport à l'espace (Rose, Tolia-Kelly, 2012). 
3 Pour aider nos lecteurs à situer cette auteure finalement assez peu connue du public français, pourriez-vous nous rappeler quelques éléments clefs de son parcours, de la place qu'elle occupe dans la littérature américaine?

4 - Jean-Marc Victor (JMV). La vie de Welty couvre la quasi-totalité du $\mathrm{XX}^{\mathrm{e}}$ siècle américain : 1909-2001. Issue d'une famille blanche aisée installée à Jackson, capitale du Mississippi, elle dit d'elle-même qu'elle mène une existence protégée, sans pour autant s'abstraire des grands tumultes de son temps, en particulier la crise de 1929, dont elle photographie les effets dévastateurs dans son État natal (elle commence à s'exprimer comme photographe avant même de publier ses premiers textes), puis la seconde Guerre mondiale, qui tient éloignés d'elle plusieurs de ses proches, puis le Mouvement des Droits civiques qui agite le Sud des États-Unis où elle passe la plus grande partie de sa vie. Elle occupe une place de premier plan dans la fiction américaine de l'après seconde Guerre Mondiale, en digne héritière des grands auteurs modernistes qu'elle admire, au premier rang desquels figurent Virginia Woolf, mais aussi, plus proche d'elle, William Faulkner, dont la formidable présence éclipse parfois les autres écrivains $\mathrm{du}$ Sud qui sont ses contemporains, comme Welty elle-même. Il ne fait guère de doute que le talent particulier de Welty pour la forme brève, même si elle fut aussi romancière, essayiste et critique littéraire, la place parmi les nouvellistes les plus remarquables du $\mathrm{XX}^{\mathrm{e}}$ siècle. Nombre de nouvellistes contemporains de langue anglaise, souvent des femmes d'ailleurs, la citent volontiers comme modèle aujourd'hui encore : Alice Munro, Joyce Carol Oates, entre autres.

5 - SW. Eudora Welty est souvent cataloguée comme un écrivain du Sud. Qu'a-t-elle de spécifique par rapport aux autres écrivains du Sud?

6 - JMV. Je propose de partir du constat qu'il existe chez Eudora Welty deux tendances contradictoires dans ce qu'elle écrit sur le Sud. Il faut dire qu'elle écrit dans le Sud sur le Sud. Elle n'a pas a priori le besoin de s'extraire du lieu pour en parler, contrairement à d'autres écrivains dont l'ancrage régional est également fort. Je pense à sherwood Anderson, dont la fiction a pour cadre le Midwest, ce qui ne l'a pas empêché de passer beaucoup de temps loin du Midwest, à Paris ou en Virginie, contrairement à Eudora Welty qui est restée quasiment toute sa vie dans le Sud. Welty écrit donc sur un territoire limité depuis ce territoire même: c'est le Mississipi et secondairement quelques États du "Vieux Sud », comme on dit. C'est dans cette zone géographique qu'elle situe la quasi-totalité de sa fiction, c'est intéressant par rapport à des auteurs qui ont ressenti plus particulièrement le besoin d'une distance.

7 Il y a un dénominateur commun aux auteurs du Sud: les plus grands, les plus connus sont plutôt en immersion. Ils peuvent avoir un mouvement de recul mais cette immersion contraste avec le contenu d'une partie de la fiction de Welty. Bon nombre de ses personnages, en tout cas les plus rebelles, qui sont souvent ceux qui lui tiennent le plus à cœur, sont plutôt dans un désir d'éloignement, d'exil, de recul, pour revenir éventuellement vers le sud afin d'y trouver cette juste distance pour apprécier le milieu d'où ils viennent. C'est typiquement le mouvement qui caractérise la vie de l'héroïne de La Fille de l'optimiste, un des plus beaux romans de Welty.

8 C'est un peu un cliché sur Welty que de la présenter comme une espèce de Miss Marple qui n'est jamais sortie de son trou, alors qu'elle a fait une partie de ses études en dehors du Sud, fait de longs séjours à New York, plusieurs mois à San Francisco. Celle qui est devenue une vieille dame qui a toujours vécu dans le Mississipi a donc une 
connaissance du reste des États-Unis. Il faut donc nuancer ce jugement, mais en même temps, elle est en immersion, malgré tout.

10 - JMV. Welty, nous aurons l'occasion d'y revenir, n'a de cesse de faire trembler les références topographiques et spatiales, identifiables. Ce faisant elle donne le sentiment que le Sud, tel qu'on se le représente, n'existe pas, mais que le Sud n'est qu'une représentation, c'est-à-dire une forme de fiction dont sa propre fiction s'empare spontanément. C'est profondément une construction collective, avec ses pesanteurs, ses contradictions, dont elle dit qu'il est utile de connaître la mécanique interne et la topographie pour parvenir à s'en déprendre et à la remettre en question. Très souvent elle a dit son exaspération d'être placée dans une catégorie d'écrivains qui serait l'«écrivain du Sud», comme elle aurait trouvé complètement inepte d'identifier Maupassant comme " écrivain régionaliste » ou « écrivain normand ».

11 Ou alors elle se sort de cette difficulté en laissant bien entendre que pour elle le Sud n'est pas la marge d'un monde plus vaste, mais peut et doit à plein titre être reconnu comme une miniature de l'ensemble du monde. Le Sud fait monde, ce n'est pas une marge du "tout», mais une miniature du tout. En cela elle rejoint de nombreux écrivains du Sud, qui eux aussi sont exaspérés d'être catalogués " écrivains du Sud ».

La question de savoir si ces écrivains sont «du Sud», c'est pratiquement la question qu'on redoute le plus de se voir poser quand on fréquente leurs textes. On s'aperçoit qu'on travaille sur des productions artistiques portant sur une zone géographique et historique particulière. En effet, je constate que ce qui m'intéresse plus spécialement, c'est ce qui n'appartient justement pas à cette dimension proprement régionale. Au contraire, c'est ce qui rejoint des questions esthétiques, qui travaillent des mouvements artistiques autres que régionaux (que ce soit le modernisme ou le postmodernisme par exemple). Mais on est bien forcé d'admettre qu'il y a des dénominateurs communs qui ont à voir avec un sens de la culpabilité, lequel a maille à partir avec l'histoire du Sud, et qu'on pourrait appeler une tache. Cette culpabilité se manifeste de manière très diverse chez les écrivains et les artistes qui en font la toile de fond (et non le sujet) de leur création.

On est en mesure de se demander finalement ce que serait ce Sud imaginaire qui est commun à des écrivains tels que Eudora Welty, Flannery O'Connor ou évidemment Faulkner - les trois qui, à mon sens, se détachent le plus. C'est une sorte d'héritage, une manière de poser cette question : comment arrive-t-on à trouver une place dans cet héritage, à s'en défaire, à remettre en question cet héritage qu'on vous a malgré vous placé sur les épaules? L'évocation de cet héritage est sans doute beaucoup plus forte chez Faulkner que chez Welty, mais c'est bien une préoccupation commune qui anime en filigrane toutes leurs œuvres. On est amené à chercher à voir comment ils arrivent à résoudre les contradictions de ce Sud réactionnaire, qui pouvait en même temps se prévaloir d'avoir atteint un haut degré de sophistication, d'éducation, un raffinement enflé par un mythe inventé après coup, que ceux qui vivaient là voulaient voir comme une réalité. La contradiction est là, entre la tache de l'esclavagisme, du racisme, de la ségrégation, d'un côté, et de l'autre côté, le prestige perdu, l'âge d'or, parfaitement imaginé, rêvé, renvoyant à la période où prétendument les Blancs avaient eu la vie facile et prospère. Cette contradiction est insurmontable. Être un écrivain du Sud c'est essayer d'y faire face. 
14 - SW. Comment cette contradiction se manifeste-t-elle concrètement dans l'écriture, dans les procédés littéraires? Pourriez-vous nous montrer comment ces auteurs se confrontent à ce problème à partir d'exemples ?

15 - JMV. Dans les manières de représenter ces espaces-là, il y a une technique qui a été très utile aux trois auteurs en question, c'est le grotesque. Parce que le grotesque sudiste, tel qu'il se pratique dans cette littérature, même s'il est hérité d'une tradition européenne, tourne très nettement autour d'un brouillage des catégories de la représentation, justement. Le grotesque sudiste s'appuie sur des représentations de l'humain, de l'espace ou des choses, qui justement ne sont pas pures. L'humain est affublé de caractéristiques animales, végétales, mécaniques. On peut ici faire un petit tour chez O'Connor : on y trouve très couramment un personnage grotesque décrit avec des oreilles de lapin, ou bien portant une valise ressemblant à hippopotame, ou encore affublé d'un visage comme une feuille de chou. Cela traduit un désir de faire fusionner les contraires et, partant, de surmonter une contradiction. Il me semble que le grotesque devient prioritairement l'outil formel ou technique de dépassement de la contradiction inhérente à l'appartenance au Sud. Comment puis-je être la laideur et la beauté absolues en même temps et, si on se place dans le champ moral, le bien et le mal simultanément? Le grotesque ce n'est pas "transcender » - un terme que j'éviterai d'utiliser -, mais réconcilier malgré eux des termes irréconciliables.

16 Le grotesque touche des lieux aussi, même si c'est moins évident. On trouvera très souvent chez ces auteurs des lieux dont les fonctions se mélangent: une poste qui devient un lieu d'habitation pour un personnage en rupture avec sa famille (« Why I Live at the P.O. » de Welty), un restaurant de troisième zone qui fait aussi dancing et station-service («A Good Man Is Hard to Find » d'O'Connor). Chez Faulkner, on trouve des lieux tout à fait hybrides, je pense en particulier à Sanctuaire, où le tripot se fait salon funéraire et où le lieu de fabrication d'alcool de contrebande est aussi celui où dort un nourrisson. Une sorte de mélange ridicule, dérisoire, de différentes fonctions : les lieux ne remplissent plus leur fonction définie initialement.

17 Au final, dans la représentation de l'espace du Sud par les écrivains « du Sud », ce qui est pris en défaut, c'est cette illusion de la possibilité d'une pureté. C'est politiquement et socialement une idée assez forte : ce qui a animé une partie de l'histoire du sud, c'était de maintenir ce qui était vu comme une pureté raciale, érigée en une sorte de mythe. Toutes ces techniques formelles qui ont à voir avec le mélange sont des réponses artistiques et pratiques, pragmatiques pour les écrivains, à cette illusion de pureté. Les formes artistiques les plus développées dans le Sud ont à voir avec l'hybridité, le mélange des catégories, la comédie est toujours une tragédie, le beau et le laid sont indiscernables. Une grande partie de ce qui s'écrit dans le Sud entre 1920 et 1960 (je m’en tiens à la période que je connais le mieux), ce sont des «tragi-comédies ». Comme le dit O'Connor dans sa correspondance : «tout ce que j'ai écrit de drôle est plus terrible que drôle, ou n'est drôle que parce que c'est terrible, ou n'est terrible que parce que c'est drôle ».

18 On est face à ce que j'aime appeler une déségrégation des genres, des formes. En mêlant ce qui est traditionnellement séparé, on produit une réaction.

19 - SW. Est-ce que cette déségrégation est liée au tournant historique de la fin de la ségrégation raciale? 
- JMV. Oui, il y a un exemple chez Flannery O'connor, dans une nouvelle intéressante de ce point de vue, "Everything That Rises Must Converge ». Dans cette nouvelle, dont l'action a lieu juste après la fin de la ségrégation, dans une grande ville du Sud, une mère vieillissante demande à son fils qui est déjà un adulte de l'accompagner dans le bus pour faire le voyage lui permettant d'aller suivre sa cure d'amaigrissement. Précisément parce que la ségrégation a pris fin dans les transports publics, elle ne veut pas voyager seule (elle est blanche évidemment). L'essentiel de la nouvelle porte sur le trajet en bus, où le jeune homme professe une grande ouverture d'esprit progressiste, se déclare antiraciste, et semble ravi de voir sa mère dans cette situation de détresse ou d'angoisse parce qu'elle est confrontée à la population noire qu'elle souhaite éviter et dont la proximité lui fait peur. L'événement unique de la nouvelle, très courte, est le suivant : une femme noire monte dans le bus accompagné d'un petit garçon qui s'assied à côté de la mère blanche, attendrie par sa présence, et alors que personne ne demande rien à personne, lui fait subitement l'aumône d'une pièce. La mère du petit garçon noir lui colle alors un coup de poing magistral dans le nez. Choqués, la mère blanche et son fils redescendent, elle fait une sorte d'attaque et reste sur le point de mourir sur le trottoir quand la nouvelle se termine. Ce qui est intéressant, c'est la manière dont une série de préconceptions dictent les conduites des uns et des autres. La mère reproduit une hiérarchisation imaginaire sur la classe et la race qui ne sont pas les siennes: le Noir doit à ses yeux se trouver dans une situation de dépendance censée marquer son infériorité. Inversement, le geste de l'aumône est vécu comme une insulte suprême pour la mère noire. Et encore à l'inverse, alors que le fils blanc nous est présenté avec des idées progressistes, si l'on explore un peu l'intérieur de ses pensées, il s'avère qu'il n'est prêt à se mêler aux Noirs que s'ils ont l' air de ministres, de professeurs ou d'avocats. À un moment, la mère dit assez clairement que les sangs mêlés sont les plus pathétiques, elle trouve leur sort tragique car ils ne sont "qu'à moitié blancs ». Elle sous-entend que chacun devrait rester de son côté de la barrière. D'ailleurs, la conception de la nouvelle se fait le miroir de ces préoccupations; de même qu'ils sont embarqués dans un bus où les catégories sont mélangées, O'Connor embarque son lecteur dans cette forme très resserrée, à l'image de l'espace restreint du bus, où le grotesque (le coup de poing est ridicule), est exposé à un moment tragi-comique saisissant.

21 - SW. Y a-t-il un attachement particulier des personnages de la fiction aux lieux qui font ce Sud tel qu'ils se les représentent ?

22 - JMV. Chez Welty, l'une des problématiques essentielles serait plutôt de savoir comment quitter le Sud, comment s'en détacher. Le Sud, c'est l'endroit d'où l'on veut partir, pour les personnages en rupture avec cette communauté, ses codes, ses pesanteurs. Il y en a quand même qui y arrivent, mais en général, ils échouent à partir. L'idée du départ est formulée de deux manières.

23 L'espace du Sud est l'endroit d'où partir, précisément dans les deux sens du terme de cette expression. Du point de vue de l'écrivain, le Sud est le point de départ de la fiction; et du point de vue des personnages, il s'agit littéralement de s'en extraire. Plusieurs personnages tentent de le faire et y parviennent parfois, mal ou pas du tout. Un seul y parvient réellement dans Les Pommes d'or, une autre est sur le point de le faire à la fin du livre tandis que le personnage quasi-mythique qui a passé sa vie à fuir revient finalement y passer ses vieux jours. C'est aussi la forme hybride de ce cycle de nouvelles qui nous alerte : il existe une seule des sept nouvelles qui ne se passe pas à 
Morgana, mais à San Francisco, le personnage ayant quitté Morgana pour San Francisco où il s'est marié. C'est aussi un élément intéressant dans le cycle: on découvre qu'il revient par la suite à Morgana, mais il y a à l'intérieur du recueil quelque chose qui n'a rien à faire là, qui ne se passe pas à Morgana. C'est là de nouveau la manifestation d'un corps étranger à l'intérieur du recueil, non pas seulement en tant que désir de quitter la ville, mais en tant que manifestation de l'hybridité du cycle. C'est une manière de montrer comment le cycle accepte de se faire pénétrer par un corps étranger. C'est la scène inaugurale de la nouvelle, une des plus magistrales des scènes d'ouverture : ils sont en train de prendre paisiblement leur petit déjeuner, les toasts grillent, d'un coup il se lève, lui flanque une gifle et s'en va. Une scène hallucinante. Le personnage, une fois sorti, au lieu d'aller travailler, décide d'errer dans les rues de San Francisco, et il tombe au hasard sur un artiste guitariste espagnol, qu'il se trouve avoir entendu en concert la veille avec sa femme. Il le suit, et comme le guitariste ne parle pas anglais et le personnage ne parle pas l'espagnol, ils communiquent par signes, et déambulent dans les rues de la ville. La scène clef de cette nouvelle très étrange, inclassable, intitulée " Musique d'Espagne ", se déroule ainsi : les personnages côtoient beaucoup d'immigrés, des Philippins, des Asiatiques plus généralement, et se retrouvent à la fin au bord d'une falaise, dans une scène à forte coloration homoérotique. Ils se donnent une étreinte très forte, on ne sait pas si cette scène est une sorte d'étreinte amoureuse ou si elle manifeste le désir diffus chez chacun des personnages de se débarrasser de l'autre, alors qu'ils sont en équilibre instable au bord de la falaise. Juste à ce moment, le personnage principal a l'impression que pénètre à l'intérieur de sa bouche quelque chose d'énorme qu'il n'arrive pas à avaler, et Welty se garde bien de commenter cette image. Ce quelque chose de radicalement étranger et autre envahit le corps. C'est intéressant que ce soit dans cette nouvelle-là, qui est le corps étranger qui envahit toutes les autres, que ce soit entre deux hommes, d'abord parce que c'est une déviation par rapport à une norme sexuelle, mais c'est aussi troublant parce que cela confirme une lecture plus érotique. Il faut ajouter que Welty a vécu une histoire d'amour douloureuse avec un homme dont elle a découvert plus tard qu'il était homosexuel, à San Francisco précisément, et elle fait état du sentiment d'exclusion qu'elle a ressenti quand elle l'a découvert.

Se mêle à cela la présence des immigrés et de cet artiste espagnol qui ne parle pas anglais, dans un univers parfaitement étranger pour lui. Cela crée un effet d'enchâssement d'une chose dans une autre à plusieurs niveaux.

\section{- SW. Existe-t-il chez Welty des espaces privilégiés chargés d'une fonction ou d'un} sens plus particuliers dans sa représentation du Sud?

- JMV. Dans Les Pommes d'or, à l'exception de la nouvelle « Musique d'Espagne », qui est la seule à ne pas se situer à Morgana, il apparait énormément de jardins. L'espace de Morgana est quadrillé par des jardins, en plus des repères topographiques, qui ont pour fonction de surdéterminer la représentation de l'espace. Les jardins sont contigus : il n'y a généralement pas de barrière entre les jardins aux Etats-Unis. Pour autant, ils définissent vraiment un espace quadrillé : on sait quel jardin appartient à untel, lequel à tel autre. C'est un quadrillage très perceptible, où dominent les blancs riches (le propriétaire du journal, le boutiquier le plus prospère...): ces jardins qui surdéterminent l'espace sont tout le temps traversés, pénétrés par des personnages qui 
n'ont rien à y faire, qui s'infiltrent en passant inaperçus et remettent en cause les limites de cet espace. Et dans l'utilisation de l'espace, non seulement ces personnages passent d'un jardin à un autre et contredisent la fixité des frontières établies, mais en plus d'eux, les plantes circulent aussi. Elles circulent de plusieurs manières chez Welty, et c'est un point qui me tient particulièrement à cœur. Beaucoup de personnages féminins sont des jardinières (Welty elle-même était une jardinière aguerrie) et beaucoup de ces femmes ont tendance à créer une circulation entre elles, par des dons de végétaux. Elles s'offrent des boutures de rosiers, des plants de tomates, des graines toujours placées sous l'auspice de la féminité. Cet échange est une manière de brouiller l'identité propre de chaque jardin : il n'existe pas de jardin parfaitement autonome mais cela circule, par ces dons féminins. On remarquera que ce sont des préoccupations proches de celles des jardiniers et paysagistes contemporains: les plantes circulent toutes seules, elles se resèment, reviennent ailleurs, se propagent. Ces représentations de la circulation des plantes à l'intérieur d'un cadre et la manière dont sont décrites les frontières non tangibles mais réelles entre les jardins, c'est très proche de ce que décrit Gilles Clément, notamment dans Éloge des vagabondes (2002), où le paysagiste évoque la liberté de circulation des plantes mais aussi très explicitement des humains, et constate qu'on n'arrêtera pas la circulation du vivant. Dans cette représentation de l'espace de la petite ville, sont dépeintes aussi bien la norme que la contestation de la norme. Welty opère une destruction du cadre et nous laisse bien voir que ça circule. Évidemment, qui se promène d'un jardin à l'autre ? Ce sont prioritairement des personnes qui sont en rupture avec l'ordre établi. L'une de celles qui traversent les jardins se trouve être la fille d'une des principales jardinières de Morgana, nommée Virgie (on pourrait proposer bien des interprétations du choix de ce prénom). Elle va et vient d'un jardin à l'autre, vole des fleurs, s'introduit même par effraction dans une maison vide pour flirter avec son petit ami. À la fin du cycle, elle décide de quitter Morgana, un geste qu'on pourrait voir comme une forme extrême de cette libre circulation. contradictoire d'ancrage et de désancrage. D'une part, le lieu tient son nom de la famille Morgan, qui est l'une des plus influentes dans la mythologie collective de la ville. Mais d'autre part, il y a un puissant pouvoir de suggestion linguistique opéré par ce nom qui évoque un mirage dans l'expression «fata morgana ${ }^{1}$ ». Le nom même de Morgana renvoie donc à la fois à un ancrage identifiable et à une illusion d'optique. On peut faire l'hypothèse que c'est la métaphore d'un ordre, d'une communauté, d'un système, qui est en fait un mirage. En nommant le lieu, Welty l'ancre dans une réalité sudiste, en disant en même temps que ça n'existe pas, que c'est une construction, un mirage, quelque chose qu'on a envie de voir, une autre fiction à l'intérieur de la fiction.

31 C'est pour ça qu'elle est politique sans l'être : elle ne dit jamais ce qu'il faut penser, ce qu'il faut croire, mais elle est spécialement, plus qu'O'Connor, sur un territoire de la suggestion et de l'oblique, en aucun cas dans la doxa.

32 - SW. Welty utilise également la carte comme procédé de pacte avec le lecteur pour situer ses fictions. Que pourriez-vous nous en dire?

з3 - JMV. Welty a dessiné pour deux de ses romans des cartes de situation du déroulement de l'action. En cela, elle n'est peut-être pas novatrice, même si ce n'est pas une pratique commune: Faulkner l'a fait avant elle. Il a cartographié son espace imaginaire 
concrètement, avec plusieurs cartes, parfois insérées, parfois pas, dans son grand cycle romanesque, tous les romans étant reliés les uns aux autres.

Ces cartes sont particulièrement intéressantes. Parlons-en plutôt chez Faulkner, où le cas est plus complexe. Les cartes de Faulkner sont très précises. Il place sur ces cartes les lieux où se déroule l'action du ou des romans, mais aussi les titres de certains romans ou nouvelles, qui se déroulent dans ce même territoire fictif - c'est un comté fictif nommé Yoknapatowpha. Faulkner superpose sur ces cartes fictives des villes ou des lieux réels et des lieux inventés par lui. Ces deux réalités se chevauchent, ce qui donne deux espaces qui se confrontent. Il a créé une petite ville du sud, un équivalent du Morgana de Welty si l'on veut, qu'il a nommé Jefferson, fondé absolument sur un modèle existant, la ville d'Oxford, où il vivait dans le Mississipi, alors que sur la carte elles apparaissent toutes les deux, distantes et distinctes l'une de l'autre dans une sorte d'effet de décollement de la fiction par rapport au réel. Ce désir de faire coexister sur le même support l'espace et le texte produit un effet proche de ce qu'on trouvera chez Welty: une tension entre un ancrage réaliste et un désancrage de la fiction, deux régimes de réalité. C'est une manière de dire que le réel qu'on croit connaître, celui d'Oxford, n'a pas plus de réalité que son équivalent dans la fiction. C'est par le biais de la carte une manière de placer sur le même plan ces lieux, pour dire qu'ils ne sont que des constructions, des représentations, un signe qui va dans ce sens-là. Le fait que ce qui sert de support à la carte et au texte de fiction, c'est la page imprimée, a aussi son importance. Faulkner et Welty créent vraiment pour le lecteur un effet de contiguïté entre fiction et carte. Puisque tout ça est imprimé en contiguïté, la fiction est un territoire, de même que la carte est un récit, une invitation au parcours sur le mode de la lecture. Cela veut dire qu'on a besoin d'un partenaire à l'autre bout, on a besoin du regard de celui qui lit, qui soit prêt à recomposer avec l'auteur le territoire en question. Au-delà de l'usage des cartes, et pour en revenir plus spécifiquement à Welty, il faut souligner la précision mathématique presque obsessionnelle qui se manifeste dans ses textes eux-mêmes pour situer les choses l'une par rapport à l'autre, entre telle ville imaginaire et telle ville réelle, (par exemple San Francisco, ou encore Vicksburg, une ville incontournable dans l'histoire de la Guerre de Sécession, très marquée par le siège de l'armée confédérée). Elle précise le kilométrage, le nombre de ponts à franchir, et fait preuve d'une précision arithmétique à placer les choses les unes par rapport aux autres en même temps qu'elle semble vouloir détruire ces repères eux-mêmes en soulignant leur nature illusoire, leur nature de "mirage " à l'image de l'illusion d'optique qu'est Morgana. Tout localiser pour mieux faire vaciller les repères. Elle situe les lieux les uns par rapport aux autres de la même manière qu'elle situe les groupes sociaux et raciaux les uns par rapport aux autres, mais ce qui l'intéresse, c'est la manière dont on navigue, dont ces groupes et localisations sont des constructions instables et poreuses. Les personnages naviguent d'une position à l'autre.

- SW. Pourriez-vous entrer un moment dans le détail des procédés littéraires que Welty utilise pour affronter des questions relatives à la représentation de l'espace et qui pourraient éclairer les questions que se posent les géographes ?

-JMV. La question de la distance est traitée, chez Welty, de manière assez photographique. Avant que d'être écrivain, et tout au long de sa carrière d'écrivain, elle était aussi photographe. La question de la distance à partir de laquelle on juge opportun d'observer ce qu'on photographie se pose à tous les photographes : de quel point de vue, d'où est-ce qu'on regarde? 
38 Elle dit que si elle parle du Sud, c'est parce qu'elle n'a pas d'autre choix que de parler de ce qu'elle connaît. Le Sud est un donné qui la place dans une position qui s'apparenterait à d'autres auteurs, par exemple des français comme Maupassant qui parle de la Normandie parce que c'est une donnée immédiate de son environnement.

39 À force de fréquenter ses textes, je dirais qu'elle a généralement une certaine tendance à se donner pour plus intuitive et moins théorique qu'elle ne l'est réellement.

40 - SW. Et l'espace? Quel traitement de l'espace, du lieu, des distances géographiques?

41 - JMV. À propos de l'espace, elle a une formule intéressante qu'elle a souvent utilisée, qui ne concerne pas le Sud spécifiquement mais le lieu (place) : "place is a confiner and a definer of what I'm doing» (« le lieu est une délimitation et un déterminant de mon travail»). Cela lui fournit un cadre. C'est assez photographique encore une fois, le confiner, qui pose des limites au territoire de la fiction : elle considère que l'espace qui l'entoure va lui fournir le cadre. Et le definer, c'est un élément de détermination, quelque chose qui détermine un certain type de contenu narratif. Comme Maupassant parlait des paysans et des bourgeois de la campagne normande, elle parle de la petite ville du sud des États-Unis, du rapport entre Noirs et Blancs, des fermes, des paysages et des traces dans le paysage qu'a laissé une histoire longue, complexe et conflictuelle.

Il y a deux tendances un peu contradictoires dans son approche de l'espace : elle a tendance d'une part à aller du côté d'une sorte d'hyper-référence sur un versant plutôt réaliste. Lorsqu'elle parle des lieux, elle est extrêmement exacte, d'une manière qui confine à l'obsession : elle dresse un relevé topographique très précis et méticuleux des petites villes qu'elle décrit. Il est très facile de se représenter l'espace rural ou celui de la petite ville à partir des détails qu'elle fournit dans sa fiction, et on voit le texte suivre l'espace : la rue principale, le square central qu'on trouve en général dans ces villes, où trône la statue du soldat confédéré, bordé par des boutiques aux façades alignées et aux toits très plats. Elle décrit tout de suite des repères immédiatement identifiables de représentation qu'elle ne crée pas, le tribunal, la prison, les églises, l'école, la gare ; on ne peut pas dire que ce sont des stéréotypes, car ils représentent effectivement le Sud, mais ce sont quand même quasiment des stéréotypes. Elle insère donc dans l'espace fictionnel des éléments réalistes dont on peut avoir l'impression qu'elle les reproduit à l'identique.

43 Cette tendance pourrait donner au lecteur l'impression de reconnaître immédiatement les lieux, créant pour lui un sentiment de familiarité. Cette familiarité peut avoir plusieurs raisons d'être au moment de la réception : le lecteur originaire du Sud les reconnaît immédiatement, celui d'autres régions des États-Unis aussi car il a eu accès à une masse de documents sur les bourgades et paysages du Sud. Il faut rappeler ici un élément important : la documentation photographique du Sud a été fortement incitée dans les années 1930 autour de la FSA (Farm Security Administration), sorte d'équivalent des missions photographiques en France, en particulier dans les zones défavorisées et rurales. La FSA a été créée par l'administration Roosevelt pour faire un état des lieux et remédier aux difficultés sociales et économiques de ces États du Sud particulièrement défavorisés et touchés de plein fouet par la grande Dépression comme le Mississipi. La FSA a constitué un fonds iconographique d'une ampleur exceptionnelle qui a été si largement diffusé dans les années 1930 et 1940 qu'il appartenait (et appartient toujours), je ne peux pas dire à l'imaginaire collectif, mais à une représentation visuelle du Sud répandue dans tous les milieux sociaux, qui s'alimente 
de ce fonds photographique, un des plus connus des stocks d'images représentant l'espace américain. Welty elle-même y a participé, elle a été embauchée comme photographe par une institution publique de même nature nommée WPA, et s'est promenée son appareil en bandoulière dans tout le Mississipi, les petites villes, les campagnes (Photographs, 1989).

Le lecteur, qu'il soit familier du Sud ou non, a donc des images préconçues formées à partir de sa propre connaissance ou de cette familiarité avec ce fonds iconographique populaire.

- SW. S'est-elle inspirée de ces photographies pour les descriptions de lieux dans ses nouvelles?

- JMV. On trouve en effet quelques exemples isolés où il s'avère qu'elle s'est inspirée de ces photos, mais c'est marginal.

\section{- SW. Et la deuxième tendance?}

Contre cette première tendance à ce qu'on pourrait appeler pour aller vite un certain réalisme, s'exerce une pression inverse dans sa fiction: elle va pervertir un certain nombre de représentations stéréotypées de l'espace et produire ce que j'appellerais des effets de désancrage. Ce qui paraît parfaitement localisé se met à flotter, brouillé dans ses repères habituels.

49 Elle s'attaque en particulier à des lieux emblématiques - on peut d'ailleurs utiliser la notion de topos, au sens premier et dans un sens plus symbolique - à des éléments qui sont en eux-mêmes des représentations de l'espace très chargées d'un passé culturel et historique, en particulier le porch et le cimetière.

50 Le porch est un terme difficile à traduire, entre la véranda et le balcon, il s'agit de cette galerie extérieure très connue aux États-Unis dans l'architecture vernaculaire en bois. Welty y situe un grand nombre de scènes, qui sont souvent des scènes clefs de sa fiction.

51 Le cimetière est un lieu où s'ancre évidemment une tradition familiale, historique, il est un repère important de la culture «sudiste » (si ce terme a un sens), un élément du décor qui rattache chaque individu à un passé.

Elle choisit précisément ces lieux archi-identifiables et prévisibles, qui participent de l'ancrage réaliste et elle les pervertit d'une manière intéressante.

53 Le porch, dans la vie quotidienne et l'architecture, est le lieu de l'échange et du contact entre le domestique et le social, entre l'intime et le public, un lieu de fluidité sociale qui permet de faire fonctionner à plein cette société du Sud dans l'espace. Welty fait des choix justement radicaux en choisissant pour décor ce porch dans des scènes où c'est tout le contraire d'un échange qui se produit. Le porch devient le lieu d'une grande indétermination factuelle. Par exemple, dans la nouvelle "Shower of Gold", un des personnages centraux de cette nouvelle et du recueil Les Pommes d'or, King, qui a disparu depuis longtemps, revient faire une visite à sa femme qu'il a abandonnée depuis un moment. Il se présente donc sur le porch de la maison. Le problème c'est qu'au moment où il revient, elle n'est pas disponible, elle est occupée dans la maison et elle ne le voit pas. Il n'y a pas de témoin qui soit absolument sûr pour attester de la présence effective de King : il n'y a que ses enfants, des jumeaux, qui disent bien avoir vu quelqu'un, mais n'ont pas reconnu leur père parti depuis si longtemps - d'ailleurs, peut-on les croire tout à fait car ce sont des enfants? - et un vieux jardinier noir, qui dit avoir aperçu King, mais avec de nouveau une impossibilité à prouver. Bien que 
beaucoup de membres de la communauté blanche dominante certifient que c'est un Noir auquel on peut faire confiance, le simple fait de le dire montre que la question de la validité de son témoignage se pose pour eux. La manière dont le récit de cet événement est fait rend impossible d'être absolument certain qu'il ait eu lieu. Alors même qu'on sait précisément où on est, qu'on connaît la fonction du lieu où il est censé s'être produit, le déposséder de ses propriétés, qui relèvent normalement d'une interaction entre le dedans et le dehors, crée une désorientation totale.

La Fille de l'optimiste est un roman qui commence par une scène fondatrice sur le porch. Le personnage, un juge vieillissant, explique les troubles de la vue qu'il a commencé à avoir pour la première fois sur son porch. Il décrit assez précisément les lieux, avec la topographie classique (tribunal, maison des voisins), et là, tout d'un coup, il a des espèces de flashes qui signent le début de sa maladie - laquelle entraînera bien des péripéties par la suite, et finalement sa mort. Le porch n'est plus le lieu de l'échange fluide et harmonieux, mais encore une fois un lieu de perturbation.

En faisant usage de ces lieux archi-normés, Welty a une manière de contester la pérennité des repères dans cet espace, un espace qui a beaucoup à voir avec un ordre établi. Si le porch est ce lieu d'échange, il est aussi implicitement un lieu de frontière, c'est un espace transitoire, entre ce qu'il est décent de montrer et indécent: de l'intime, du personnel, par rapport au groupe.

Pour le cimetière, ce n'est pas exactement la même chose. Pour situer l'importance du lieu, il y a une expression courante dans le Mississipi : au lieu de demander « where are you from?", on demande "where do you bury?" ("où enterrez-vous vos morts"?). Welty a d'ailleurs consacré tout un livre à des photographies prises dans les cimetières du Mississippi (Country Churchyards, 2000). Pour donner un exemple de pervertissement de la fonction du cimetière, on peut revenir au roman La Fille de l'optimiste et au recueil Les Pommes d'or, qui comportent des scènes d'enterrement, avec des détails un peu compliqués, mais dans les deux cas, les personnages qui viennent enterrer les morts sont désorientés dans les cimetières qu'ils n'arrivent pas à reconnaître. Ils sont troublés par leur chagrin, en arrivent à confondre les tombes, se demandent même si certaines personnes sont encore vivantes ou mortes, si elles ne les trouveraient pas là alors qu'elles sont vivantes... Cette fonction normée du cimetière ("voilà d'où on vient ») est pervertie. Dans Les Pommes d'or, un personnage est ostracisé tout au long du recueil. C'est une professeure de piano d'origine allemande, Miss Eckhart, qui est stigmatisée par la communauté comme plus ou moins folle, incontrôlable, étrange, d'une culture autre. Elle assiste à l'enterrement de l'homme qu'elle a toujours aimé en secret. Le rituel se déroule de manière très contrôlée par la communauté et tout à coup, elle veut se jeter dans sa tombe. Les autres la retiennent et l'en empêchent. Par ce geste, elle essaie de trouver sa place dans le cimetière, la place qu'elle n'avait pas dans la communauté des vivants, une place qui pourrait rejouer ce désir d'ancrage, d'être quelque part, à l'encontre de la communauté qui lui interdit cette place.

57 - SW. Welty fait donc jouer un rôle à la figure de l'étranger, de l'altérité ?

58 - JMV. Le rapport à l'étranger est en effet omniprésent. Un des verbes les plus récurrents dans la rumeur de la petite ville, dans ce discours anonyme de la communauté, par de petites incises dans le cours du récit, c'est le verbe «to place » qui dit à la fois où on se situe, de quelle famille, ville ou village on vient. To place: situer, dans les deux sens du terme, spatial et social, ce désir devient une véritable obsession. 
59 Là où Welty subvertit le modèle, c'est qu'elle fait le choix d'une forme littéraire hybride. La construction des Pommes d'or est voulue comme un cycle de nouvelles et non comme un habituel recueil, ce n'est pour autant pas non plus un roman. Cette organisation interne du cycle rend difficile pour le lecteur de situer le genre auquel appartient le texte. Ce n'est pas un roman, ni un recueil, c'est une collection de textes qui sont reliés entre eux, soit explicitement, soit de manière souterraine. Dans Les Pommes d'or, le lien qui se tisse d'une nouvelle à l'autre, c'est le nom de cette petite ville, Morgana, une ville fictive, et la récurrence de personnages qui reviennent, des noms des familles qui contrôlent cette communauté, qui dominent cette ville (parce qu'elles font autorité d'une manière ou d'un autre) et ce sur un laps de temps d'une quarantaine d'années.

60 Cette structure hybride vient en appui d'une contestation des normes établies, des catégories dans lesquelles doit entrer la fiction: elle constitue un entre-deux où il devient difficile de situer l'œuvre. Car finalement, que propose Welty à son lecteur qui doit relier les nouvelles les unes aux autres à l'intérieur des Pommes d'or, si ce n'est de rétablir des liens et une unité entre des éléments prétendument disjoints, épars, hétérogènes? Voilà ce qui confère l'essentiel du sens au cycle de nouvelles dans sa construction formelle bien particulière. Cela relève de la même logique d'abolition des frontières et des limites : une hybridation des textes les uns avec les autres.

61 - SW. La notion de marge est-elle importante dans la configuration de ce recueil ?

62 - JMV. Il y a très clairement une place marginale, littéralement marginale, dans l'espace à attribuer à certains personnages dans cette communauté de Morgana. Par exemple au tout début du recueil, il est question d'une description topographique de la rue principale, de maisons identifiables car appartenant à des familles connues, mais on apprend qu'« un peu plus loin », il y a l'orphelinat du Comté. C'est d'abord simplement une mention au début de la première nouvelle, mais cela revient plus tard dans une autre nouvelle du recueil. Là, Welty décrit comment cohabitent au bord d'un lac, Moonlake, pendant les vacances d'été, les enfants des principales familles de Morgana en colonie de vacances et les enfants de cet orphelinat. Au bord de ce lac qui est justement en périphérie, les enfants des classes dominantes cohabitent avec une classe inférieure (sans même parler des Noirs) : cette place est définitivement "à côté ", à la fois dans le sens d'une contiguïté et d'une marginalité par rapport à la ville ici invisible (Morgana n'apparaît pas dans cette nouvelle).

63 Je trouve important de relier toujours ce procédé à la forme que prend le recueil : c'est un recueil totalement éclaté, composé de sept nouvelles, toutes reliées entre elles, dont il est impossible de déterminer le centre. Il n'y a pas de personnage principal, pas de centre ni de périphérie dans les lieux : d'une nouvelle à l'autre, ils changent. C'est la forme même du cycle qui fonctionne comme antidote à ce désir centripète de la communauté à l'endroit des marginaux.

64 - SW. Les présente-t-elle explicitement comme des marginaux, comme dans tant de romanciers ou réalisateurs américains de cette période l'ont fait, avec la figure du hobo?

65 - JMV. "Marginaux », non, c'est excessif. Ils ne le sont que dans le regard des autres. Le texte ne leur donne pas d'essence d'une quelconque altérité, à part l'impossibilité de les placer (dans un passé ou dans une tradition). Que ce soit pour Miss Eckhart ou certaines orphelines au bord du lac de Moonlake - il est intéressant de remarquer que ce sont 
souvent des femmes - on n'a jamais accès à leur intériorité : Welty se place du point de vue de la communauté les observant. Ces femmes conservent un degré d'opacité qui n'est pas une opacité intrinsèque. Cette opacité exprime peut-être simplement une position qu'on pourrait qualifier de respectueuse de la part de Welty. Celle-ci est issue d'une classe sociale blanche privilégiée ; elle est donc dans une position délicate pour se mettre «à la place de ». Elle fait le choix de ne pas adopter le point de vue de la marge.

C'est aussi le choix d'un point de vue qui, politiquement, même si le terme " politique " est un gros mot dans son cas, va peut-être avoir un impact plus fort sur le lecteur en le mettant plus facilement dans une proximité sociale avec les observateurs. Elle fait le pari de se placer dans une position moyenne plutôt que dans la position du marginalisé.

68 - JMV. Ce point ne fait pas l'unanimité. Tous les commentateurs s'accordent sur le fait qu'il y a dans ses œuvres une charge critique sur la rigidité d'un système, depuis l'intérieur, visant toutes les formes de cette rigidité, qu'elle soit sociale, raciale ou morale. Et en même temps, il lui a été souvent reproché de ne pas être assez explicite dans ses positions. Un reproche à quoi elle a toujours rétorqué que le romancier n'est pas un "croisé", ne "part pas en croisade», et que "la netteté est le plus grand danger de la fiction ». Un de ses essais critiques les plus connus s'intitule « Must the Novelist Crusade?» (1987 [1965]), et la réponse est clairement négative. Elle n’a pas désavoué des lectures politiques de ses œuvres, mais elle ne les a ni validées, ni invalidées. Elle considère que le rôle de l'artiste est de se placer sur une ligne de frontière, de faire trembler cette frontière entre le net et le flou. Il y a un désir profond de ne pas pouvoir être assigné à une place, en particulier à la place de la militante. Y être assignée l'insupporte. On lui en a fait le reproche dans les années 1960, au moment du mouvement pour les Droits civiques. Elle a reçu beaucoup de coups de téléphone en pleine nuit qui insistaient pour lui demander pourquoi elle ne prenait pas position plus ouvertement. Elle répondait que ce n'était pas son rôle.

69 Cependant il y a un texte sur lequel il faut revenir. À la suite de l'assassinat d'un leader local du mouvement des Droits civiques, elle a écrit une nouvelle très surprenante. "Where Is the Voice Coming From » est une nouvelle écrite à la première personne, où elle se met à la place de l'assassin raciste, un obscur qui passe à l'action. On voit l'ambiguïté qu'il y a à répondre à ces attaques de cette manière : au lieu d'une prise de position explicite, elle se met à la place de l'assassin blanc, et elle nous fait partager toute l'horreur, la violence, l'extrémisme du discours raciste, avec une infinie froideur qui est déstabilisante à la lecture. Encore une fois, elle ne choisit pas le point de vue le plus transparent, ni le plus consensuel, elle est un peu du côté du scandale, en forçant le lecteur à s'identifier à une position monstrueuse.

70 - SW. Est-ce que la représentation des lieux chez Welty est comparable à ce que font d'autres auteurs, comme Flannery o'Connor que vous connaissez également bien?

71 - JMV. Chez O'Connor, le type d'espace privilégié, du moins l'un des lieux les plus récurrents, c'est la ferme, le plus souvent une ferme en Géorgie, la petite propriété familiale tenue par des Blancs, souvent des femmes, en l'absence de mari, qui sont souvent absolument sûres d'elles-mêmes, convaincues d'être en haut de la hiérarchie sociale, de détenir certaines vérités sur leurs croyances, leurs pratiques religieuses, leur statut social. La ferme, qui est une miniature du modèle de la plantation, devient pour elles un trésor, une forteresse à protéger contre les intrusions du monde 
extérieur. Les stratégies de protection apparaissent au gré de rangées d'arbres qui font obstacle à l'entrée de la lumière, de l'étranger, de barrières, de clôtures... C'est un modèle de forteresse à protéger contre l'extérieur, forcément menaçant, et O'Connor s'amuse à les exposer systématiquement à des formes souvent sexualisées de pénétration ou d'envahissement. Dans la nouvelle "Greenleaf ", Mrs May, une femme qui tient une ferme seule contre vents et marées, sans mari, avec des enfants qui s'avèrent incapables, fait travailler des employés blancs et d'autres, noirs. Ses employés blancs en particulier l'indisposent car ils ont l'air de réussir mieux qu'elle et ils possèdent un taureau qui ne cesse de s'introduire chez elle et mange ses plates-bandes. La nouvelle s'ouvre sur le taureau qui est déjà entré et ravage le jardin. Mrs May n'a de cesse de réclamer aux employés blancs de l'en débarrasser, et dans la chasse au taureau qui s'en suit, elle finit éventrée par une corne, formant alors un grotesque à moitié animal et à moitié humain : le plus honni fait finalement partie d'elle, a pénétré en elle. o'Connor utilise des formes plus violentes que Welty pour montrer comment une pureté fantasmée est déconstruite comme étant illusoire, impossible, et au final pas du tout souhaitable. Tout cela s'ancre dans ce territoire de la ferme, de la barrière, de la clôture.

Toutes les deux, Welty et O'Connor, ont dit souffrir d'avoir écrit après Faulkner. Il était difficile pour elles que ce soit un homme qui ait eu un tel retentissement et que ce soit lui en particulier: il a fait énormément d'ombre aux écrivains, en particulier aux écrivaines. Ce n'est pas un rejet, elles sont très admiratives de son oeuvre, mais elles expliquent qu'il n'y avait plus beaucoup de place pour d'autres. La critique littéraire en tant qu'institution pouvait encore à la rigueur accorder une place à une exception sudiste si c'était un grand bonhomme comme Faulkner mais pas à d'obscures écrivaines provinciales. Cela confirme que le Sud est une invention: une invention des Sudistes (par leur tradition, leur mythe etc.), et une invention du Nord. On peut se référer à un texte très connu de Mencken (1917), journaliste et essayiste qui a écrit que le Sud était "the Sahara of the Bozart» ("le Sahara des Beaux-Arts»). Cette expression est une appellation qui a longtemps collé au territoire malgré la richesse de sa production artistique. Si on accepte qu'un génie ait pu surgir de ce désert, on n'en accepte pas plusieurs, et encore moins des femmes.

\section{Romans et nouvelles mentionnés dans cet article}

- Faulkner William, 1993 [1931]. Sanctuary. New York, Vintage International.

- O'Connor Flannery, 1990 [1971]. The Complete Stories. London, Faber and Faber.

-Welty Eudora, 1980 [1949]. The Golden Apples. The Collected Stories of Eudora Welty. New York, Harcourt Brace Jovanovich, p. 259-461. Traduction française : Les Pommes d'or. Trad. Michel Gresset et Sophie Mayoux. Paris, Flammarion, 1995.

-Welty Eudora, 1984 [1972]. The Optimist's Daughter. London, Virago. Traduction française : La Fille de l'optimiste. Trad. Louise Servicen. Paris, Calmann-Lévy, 1974. 


\section{BIBLIOGRAPHIE}

Bellehigue M., Victor J.-M., 2004. Quelques figures de l'abolition des frontières chez Flannery O'Connor. Études anglaises, vol. 57, $\mathrm{n}^{\circ}$ 4, p. 438-452.

Clément G., 2002. Eloge des vagabondes. Paris, Nil.

Mencken H. L., 1917. The Sahara of the Bozart. The New York Evening Mail, 13 novembre.

Rose G., Tolia-K D. (dir.), 2012. Visuality/Materiality. Images, Objects and Practices. Farnham, Burlington, Ashgate.

Victor J.-M., 2009a. Trying Words Once Again" : conjurer la chute dans "A Curtain of Green" de Eudora Welty. Revue française d'études américaines, $\mathrm{n}^{\circ}$ 121, p. 29-38.

Victor J.-M., 2009b. Regarder ceux qui regardent : Eudora Welty photographe. Transatlantica, $\mathrm{n}^{\circ}$ 2009-2, http://transatlantica.revues.org/4559

Victor J.-M., 2009c. Détours et délitements : ce qui se défait dans quelques photographies de Ralph Eugene Meatyard. Revue française d'études américaines, $\mathrm{n}^{\circ} 120$, p. 54-64.

Victor J.-M., 2010. “Les Suds profonds de l'Amérique”. Photographies de Ralph Eugene Meatyard, Clarence John Laughlin et Alex Harris. Transatlantica, n 2010-2, http://transatlantica.revues.org/ 5085

Welty E., 1987 [1965]. Must the Novelist Crusade? In The Eye of the Story. Selected Essays and Reviews. London, Virago, p. 146-158.

Welty E., 1989. Photographs. Jackson, University Press of Mississippi.

Welty E., 2000. Country Churchyards. Jackson, University Press of Mississippi.

Woolf V., « Kew Gardens ». Traduction française par Nathalie Pavec et Jean-Marc Victor. Jardins, $n^{\circ} 3,2012$, p. 49-55.

\section{NOTES}

1. Une fata morgana est une illusion d'optique résultant d'une combinaison de mirages barrant un horizon marin et causés par la superposition de couches d'air chaud et froid. Ce phénomène est visible dans certaines mers. Son nom révèle qu'il a été attribué dès le Moyen-Âge à la fée Morgane, qui dans la légende arthurienne avait le pouvoir de bâtir des palais sur les eaux.

\section{AUTEURS}

\section{JEAN-MARC VICTOR}

Jean-Marc Victor est maître de conférences en études américaines, Université Paris 4 Sorbonne. 
SERGE WEBER

Serge Weber est maître de conférences en géographie, urbanisme et aménagement, Université Paris-Est Marne-la-Vallée. 